\section{THE USE OF OPTICALLY TRIGGERED, HIGH GAIN GaAs SWITCHES FOR UWB PULSE GENERATION}

\author{
G. M. Loubriel, F. J. Zutavern, M. W. O'Malley, \\ R. R. Gallegos, and W. D. Helgeson
}

High Power Electromagnetics Department Sandia National Laboratories

Albuquerque, NM 87185-1153

\begin{abstract}
A high peak power impulse pulser that is controlled with high gain, optically triggered $\mathrm{GaAs}$ Photoconductive Semiconductor Switches (PCSS) has been constructed and tested. The system has a short $50 \Omega$ line that is charged to $100 \mathrm{kV}$ and discharged through the switch when the switch is triggered with as little as $90 \mathrm{~nJ}$ of laser energy. The laser that is used is a small laser diode array whose output is delivered through a fiber to the switch. The current in the system ranges from $1 \mathrm{kA}$ (with one laser) to $1.3 \mathrm{kA}$ (with two) and the pulse widths are 1.9 and $1.4 \mathrm{~ns}$, respectively. The peak power and the energy delivered to the load are $50 \mathrm{MW}$ to $84 \mathrm{MW}$ and $95 \mathrm{~mJ}$ to $120 \mathrm{~mJ}$ for one or two lasers. The small trigger energy and switch jitter are due to a high gain switching mechanism in GaAs. This experiment also shows a relationship between the rise time of the voltage across the switch and the required trigger energy and switch jitter.
\end{abstract}

\title{
INTRODUCTION
}

This research has focused on optically triggered, high gain GaAs switches for high speed, high power electronics and optoelectronics. The practical significance of this high gain switching mode is that the switches can be aclivated with very low energy optical triggers. I For example, this work will show that a $90 \mathrm{~nJ}$ optical pulse has triggered switches that have delivered $50 \mathrm{MW}$ for $-1 \mathrm{~ns}$ in a $50 \Omega$ system, and previously we have switched $6 \mathrm{MW}$ for $-100 \mathrm{~ns}$ in a $0.25 \Omega$ system. ${ }^{2}$ The GaAs switches used in this experiment are lateral switches: they have two contacts on one side of a wafer separated 
by an insulating region of intrinsic material (sec figure 1). Al electric fields below 4 $\mathrm{kV} / \mathrm{cm}$, the GaAs switches are activated by the creation of, at most, one electron hole pair per photon absorbed. This linear mode demands high laser power, and after the light is extinguished, the carrier density decays in $1-10 \mathrm{~ns}$. At higher electric fields these switches behave very differently. The high field induces carrier multiplication so that the amount of light required is reduced by as much as five orders of magnitude 1,2 . This high gain mode is characterized by fast current rise time: $(-400 \mathrm{ps})$ and filamentary currents with densities of several $\mathrm{MA} / \mathrm{cm}^{2}$ and diameters s $f 50-300 \mu \mathrm{m}$ (from the photographs of recombination radiation). In the "on" state there is a characteristic, constant field across the switch called the lock-on field. The switch current is circuit-limited provided the circuit maintains the lock-on field. ${ }^{2}$ Table 1 shows the results from this experiment and the best results that we have achieved (in other work) with the high gain GaAs switches when triggered with compact laser diode arrays. The works of many others has been presented at various conferences. 3

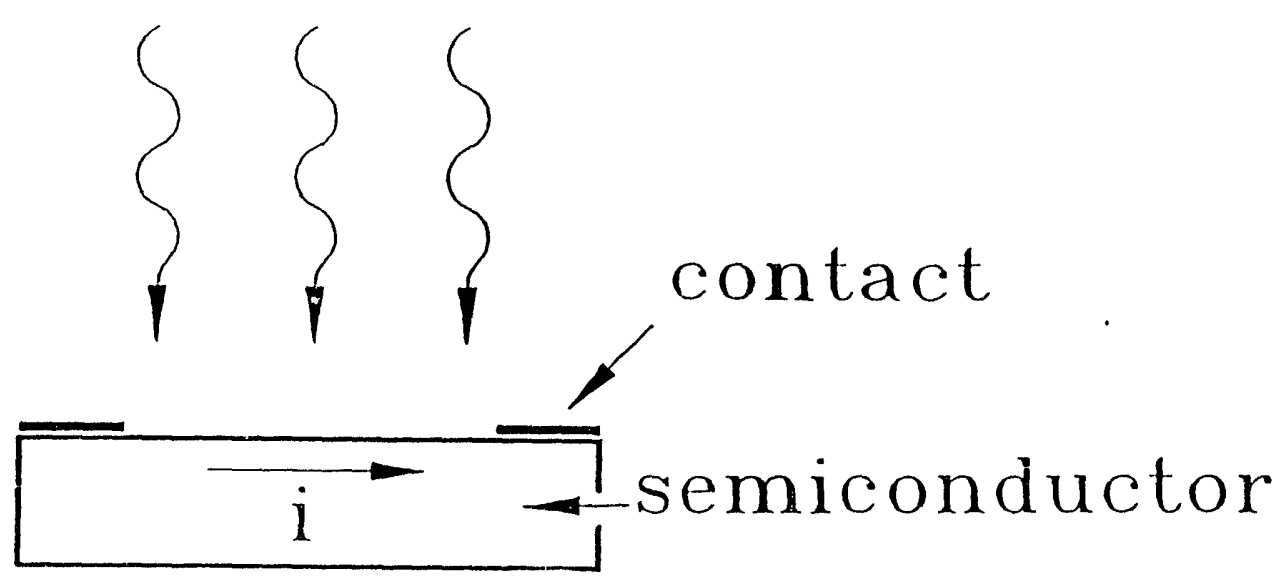

Figure 1. Schematic of the lateral switches that are used in this study. Light illumination is either uniform (as shown) or through a small fiber optic line that ends near one of the contacts.

Table 1. Summary of results from tests where high gain GaAs switches are triggered with laser diode arrays (LDA).

\begin{tabular}{|l|l|l|}
\hline & This Exp. & Other $^{*}$ \\
\hline Switch Voltage (kV) & 100 & 155 \\
\hline Switch Current (kA) & 1.0 & 5.2 \\
\hline Peak Power (MW) & 50 & 50 \\
\hline Rise time (ps) & 430 & 430 \\
\hline R-M-S jitter (ps) & 150 & 150 \\
\hline Optical Trigger Energy (nJ) & 90 & 90 \\
\hline Repetition Rate (Hz) & 1,000 & 1,000 \\
\hline Electric Field (kV/cm) & 67 & 100 \\
\hline Device Lifetime (No.pulses) & $\mathrm{NA}$ & $50(0,00)$ \\
\hline
\end{tabular}

* Not all the results are simultaneous. 


\section{EXPERIMENTAL, SETUP}

The circuit that was used in these tests is shown in Figure 2. It operated in bursts of up to 5 pulses at a repetition rate of $1 \mathrm{kHz}$. We charged a nominally $1.0 \mathrm{~ns}$ long, $47 \Omega$, parallel plate transmission line. This line is discharged with either one or two switches into a $50 \Omega$ load. We measured the voltage on the transmission line and the current through the load. A typical transmission line voltage waveform is shown in Figure 3 . The voltage on the line, shown at $100 \mathrm{~ns} / \mathrm{div}$, rose to a peak value with a charge time of $210 \mathrm{~ns}$. At this point the laser activated the switch and the line voltage drop $p_{2}$ ed. If only one switch was triggered, the resulting load voltage was a monopulse. If both switches were triggered simultaneously the load current was a monocycle (bipolar pulse). Previous studies show that, as the switched field increases, the switch rise time decreases and the trigger energy is reduced. 2 We used switches with an insulating region of $1.5 \mathrm{~cm}$ at a voltage of $100 \mathrm{kV}$. At this voltage, the energy on the charge line was $54 \mathrm{~mJ}$. Because the fields across the switch were above air breakdown the switches were immersed in a dielectric liquid (Fluorinert ${ }^{8}$ ). To avoid corona and breakdown, the transmission line was in $\mathrm{SF}_{6}$ gas.

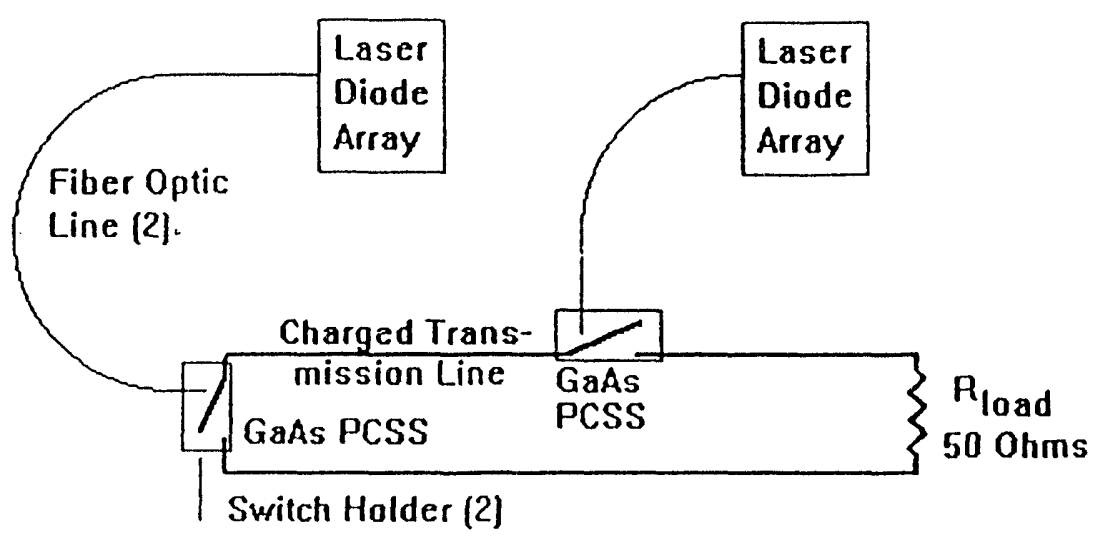

Figure 2. Schematic of the circuit that was used in these experiments. A short ( $1 \mathrm{~ns}), 47 \Omega$ transmission line (the charge line) was charged to high voltage at a burst repetition rate of $1 \mathrm{kHz}$. Two switches were used on either side of the line to discharge the line into a $50 \Omega$ load.

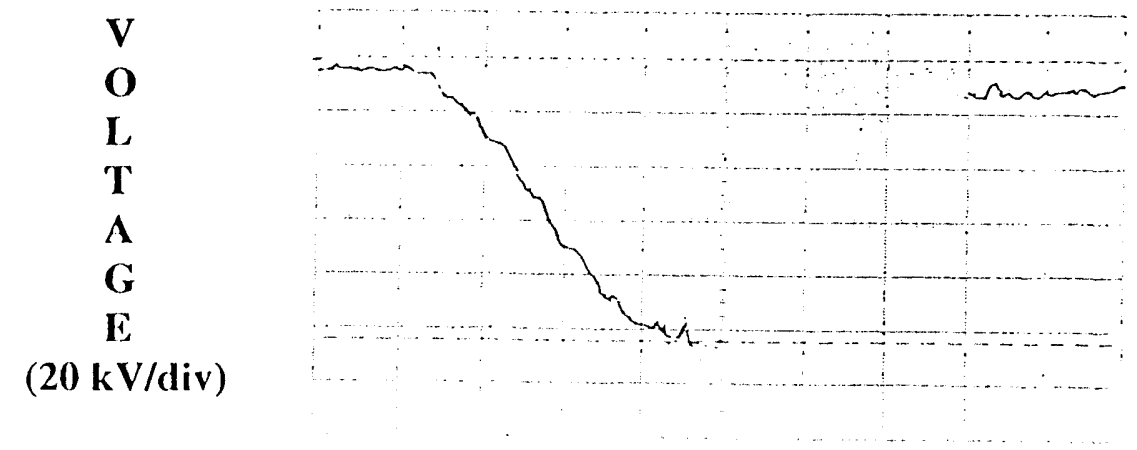

TIME (100 ns/division)

Figure 3. The voltage on the charge line. The waveform is displayed at $20 \mathrm{kV} /$ div. (0) is one division from the top) and at $100 \mathrm{~ns} /$ div. The charge time is $210 \mathrm{~ns}$ and the peak voltage is $100 \mathrm{kV}$. When the voltalye reaches its peak value of $100 \mathrm{kV}$ the laser diode arrays triggers the switch (at the center of the waveform) discharging the line. 


\section{RESUI'TS}

In the first set of tests both laser diode arrays were used to activate one switch and obtain a monopulse. The highest current measured with this system is shown in Figure 6. The width of the current pulse and its peak value depend on the time delay between when the two laser diodes are triggered. When both diodes are triggered to produce simultaneous current pulses, the current is largest and the current pulse width is smallest. The highest current (figure 6) was $1.3 \mathrm{kA}$ with a rise time of $430 \mathrm{ps}$ and a pulse width of $1.4 \mathrm{~ns}$. This corresponds to $84.5 \mathrm{MW}$ (peak) with $180 \mathrm{~nJ}$ of trigger energy. With one laser diode activating one switch the current is about $1.1 \mathrm{kA}$ with a rise time of about $770 \mathrm{ps}$ and a pulse width of $1.8-1.9 \mathrm{~ns}$.

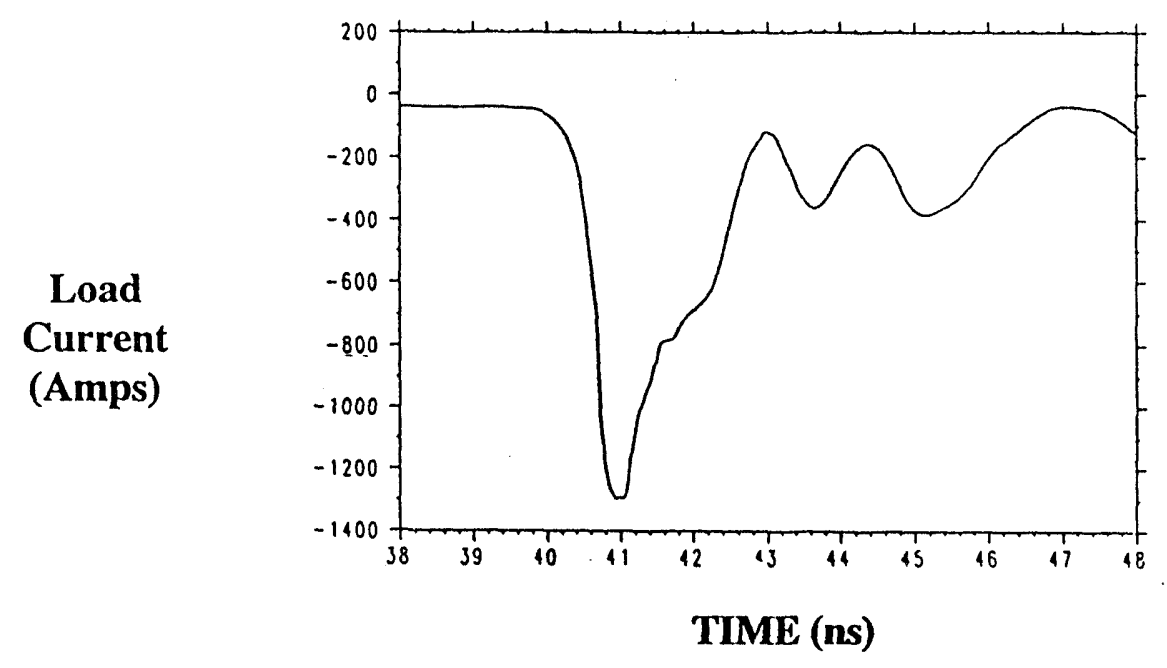

Figure 6. The current through the $50 \Omega$ load when both laser diodes are used to trigger one switch. This is the highest peak current $(1.3 \mathrm{kA})$, the highest peak power $(84.5 \mathrm{MW})$, and the smallest width $(1.43 \mathrm{~ns})$ that we measured.

The difference in current waveforms when we use one laser versus two may be due to two different reasons: a difference in the switch inductance and a the dynamics of the high gain process. Our circuit simulations show that the current risetime for a total inductance of $18 \mathrm{nH}$ would be about 430 ps with a width of $1.3 \mathrm{~ns}$. An inductance of $40 \mathrm{nH}$ results in a rise time of $740 \mathrm{ps}$ with a width of $1.6 \mathrm{~ns}$. Thus it may be possible that one filament with an inductance of $40 \mathrm{nH}$ results in one current waveform and two filaments with about half the inductance create a faster current pulse with a faster rise and smaller width. The problem with this scenario is that the inductance we expect, based on the pictures of the filaments that gave rise to these current waveforms, is much smaller. In this setup the inductance of a filament is estimated to range from $15 \mathrm{nH}$ to $21 \mathrm{nH}$ for filament radii of $300 \mu \mathrm{m}$ to $50 \mu \mathrm{m}$, respectively, assuming that the filament kecps that radius for $1.5 \mathrm{~cm}$. The pictures show a filament that starts small but ends with a width of about $1 \mathrm{~cm}$. The inductance would be much smaller $(4 \mathrm{nH})$. Thus, there are other factors contributing to the different waveforms. One possibility is that the gain in one filament may be affected by the presence of the other filament resulting in a faster process: the lower current density in each filament may allow it to create more carriers, especially if there isian upper bound in the carrier density. Another possibility is that both filaments are generating carriers and thus the time required for their combined resistance to drop from $2 Z$ 10 $7 / 2$ (where $Z$ is the impedance of the system) is reduced by a factor of two 
Two laser diode arrays were used to trigger the switches. Bach combined of three laser diodes coupled to a $300 \mu \mathrm{m}$ fiber optic. Each array delivered $9(0) \mathrm{n}$ in $+.2 \mathrm{~ns}$ at 876 and $857 \mathrm{~nm}$ to a spot near the positive high voltage $(100 \mathrm{kV})$ side of the switch. For other tests, these same laser diode arrays were configured to produce a longer pulse (20 ns) with larger energy $(1.8 \mu \mathrm{J})$ and power $(90 \mathrm{~W})$. The waveforms for the laser output are shown in Figures 4 and 5 for the $90 \mathrm{~nJ}$ and $1.8 \mu \mathrm{J}$ configurations, respectively.

All the monitors were calibrated. The calibration of the low bandwidth voltage monitor was straightforward. We assume the calculated values of the impedance of the system. The calibrations of the load resistor and current viewing resistor in parallel with it were carried out at low voltages and at low bandwidth. For this system, charged to $100 \mathrm{kV}$ with a line impedance of $47 \Omega$ and a load resistor of $50 \Omega$, the maximum current that we expect is $1.0 \mathrm{kA}$. We measured up to $1.3 \mathrm{kA}$. Because the electrical skin depth at the high frequencies is smaller than at the frequencies where the current viewing resistor is calibrated, it's resistance may be higher than measured. This affects the calibration and may be the reason why the currents are too high.

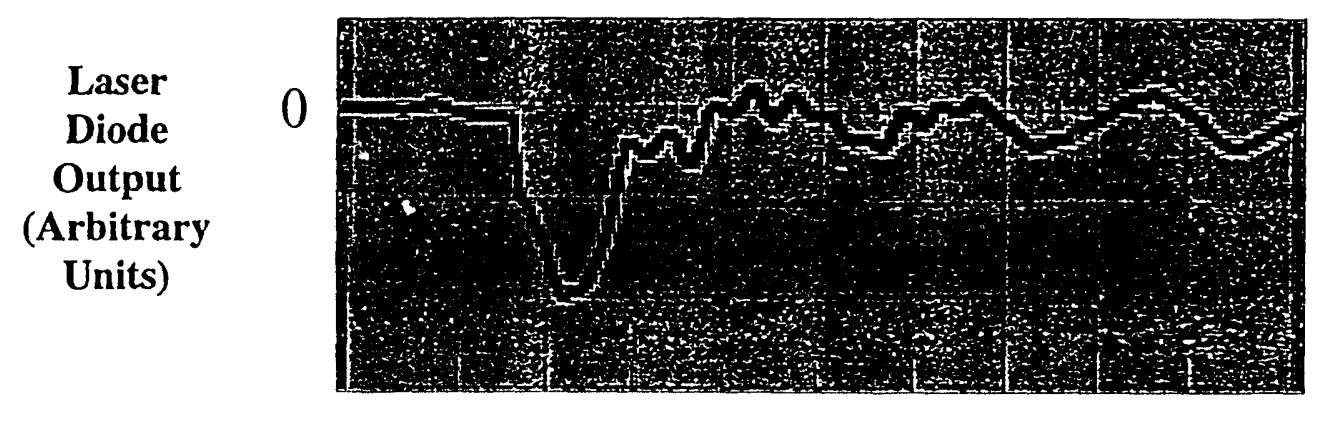

TIME ( 5 ns/division)

Figure 4. The output (arbitrary units) from the laser diode arrays configured in such a way that the total energy in the pulse is $90 \mathrm{~nJ}$. The pulse duration is $4.2 \mathrm{~ns}$. The peak power is $21 \mathrm{~W}$.

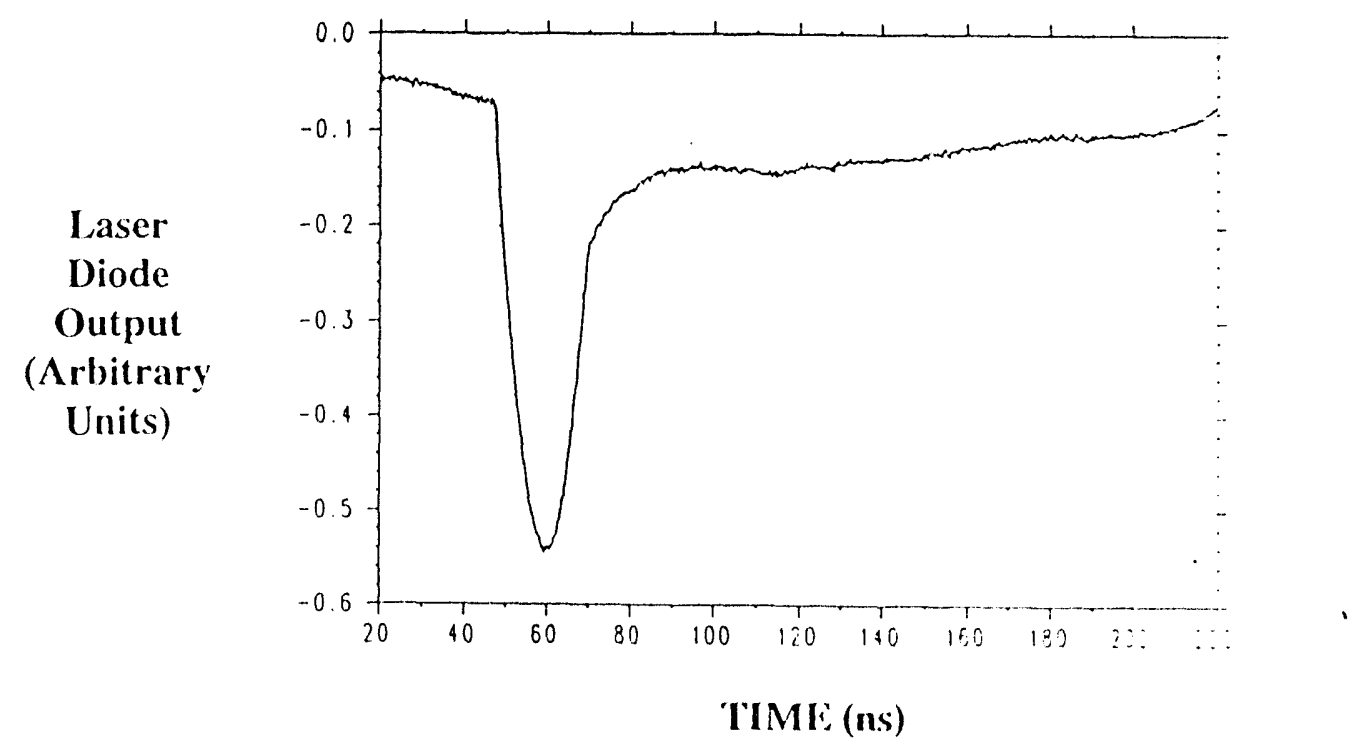

ligure 5. The output (arbitrary units) from the laser diode arrays configured in such a way that the total energy in the pulse is $1.8 \mu \mathrm{J}$. The pulse duration is about 20 ns. The peak power is 9() $\mathrm{W}$. 
The second set of tests utilized both laser diodes, each triggering one switch, to produce at monocycle. Figure 7 shows the current waveform. In theory, with ideal switching, the monocycle should be composed of two monopulses of opposite polarily each with half the pulse width. Thus, we expect a monocycle composed of a negative and positive pulses with a width (each) of 0.9 ns. What we observe is a width of 1.0 ns for the negative pulse and $1.3 \mathrm{~ns}$ for the positive pulse. The reason for this is a timing error of about $200 \mathrm{ps}$. The minimum width should occur when both switches are triggered simultaneously. It is very important to trigger both switches at the same time to obtain full voltage and to obtain the proper waveform. In our tests, the switch jitter did not allow us to always reproduce the waveform of Figure 7 even with identical starting conditions.

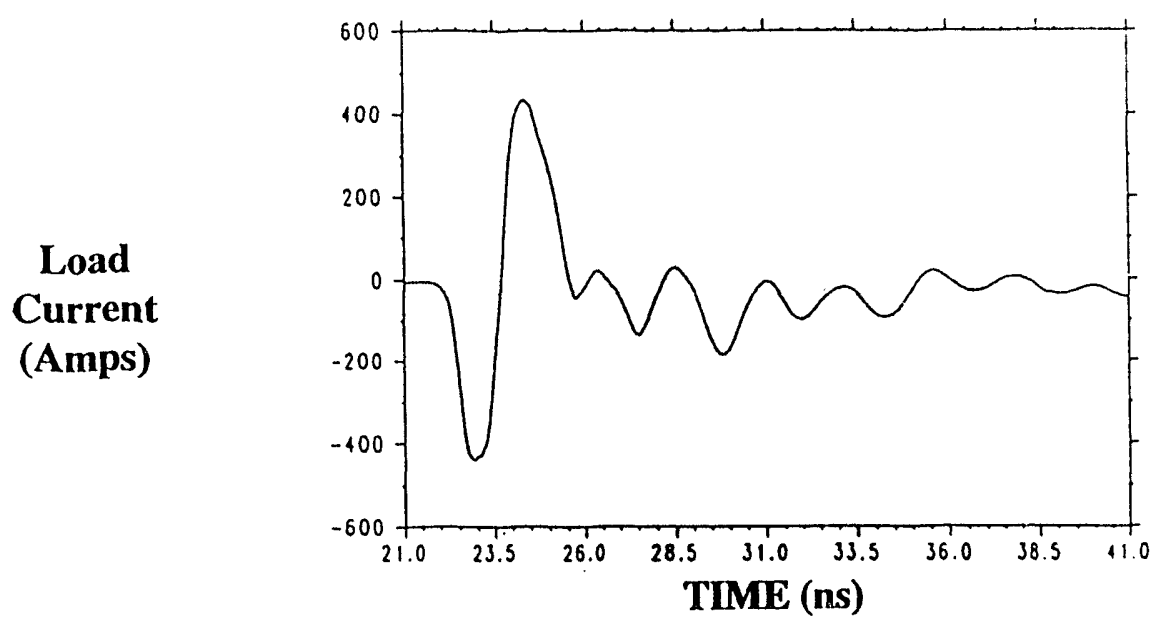

Figure 7. The current through the $50 \Omega$ load when each of the two laser diodes is used to trigger one switch.

Low jitter triggering at 90 to $180 \mathrm{~nJ}$ of optical energy depends on the rise time of the pulse charging (voltage) waveform. We tested this effect in a experiment where the first to last timing spread was recorded for different voltage rise times $(210,590$, and $865 \mathrm{~ns})$ and different laser energies $(90 \mathrm{~nJ}$ and $1.8 \mu \mathrm{J})$. Neither laser energy triggered the switch with the $865 \mathrm{~ns}$ rise time. The $90 \mathrm{~nJ}$ did not trigger the switch when the voltage rise time was 590 ns. The $1.8 \mu \mathrm{J}$ did trigger the switch when the rise time was 590 ns but only about half the time. The first to last timing spread was 6 ns for one ten pulse sequence and up to $100 \mathrm{~ns}$ in others. For the $213 \mathrm{~ns}$ rise time both laser energies resulted in timing spreads of $<1$ ns. The experiment shows a relationship between the rise time of the voltage across the switch, the required trigger energy, and switch jitter. This is in marked contrast to the switch rise time for linear photoconductivity where the drop in switch resistance is dependent only on the laser pulse and the carrier lifetime. Note that the dielectric relaxation time, $\rho \varepsilon$, is $11.6 \mu \mathrm{s}$. Thus, these effects are occurring at times that are much shorter than the relaxation time. It may be possible that the effect that we observe is related to trap filling in the GaAs because trap filling affects the electric field distribution.

\section{CONCLUSION}

This study has shown that it is possible to obtain high peak power ( $>50 \mathrm{MW}$ ) impulses in a system with an impedance of $50 \Omega$ using laser diode triggered PCSS operated in the high gain mode. The system was operated at a burst repetition rate of 1 kHz. The system is very small because laser diode arrays of very small energy output (9) nJ) were utilized (o) trigger the switches. 'The ability of the laser diodes to trigger the switches was enhanced 
by fast ( $210 \mathrm{~ns})$ charging of the transmission line which the switch discharges. An added benefit of the faster charging was a small switch jitter (150 ps). The small jitter may allow the use of these pulsers in transmitter arrays.

\section{REFERENCES}

[1] G. M. Loubriel, M. W. O'Malley, and F. J. Zutavern, "Toward pulsed power uses for photoconductive semiconductor switches: closing switches," Proc. 6th IEEE Pulsed Power Conference, P. J. Turchi and B. H. Bernstein, eds., Arlington, VA, 1987, pp. 145148.

[2] F. J. Zutavern and G. M. Loubriel, "High Voltage Lateral Switches from Silicon or Gallium Arsenide, in High-Power Optically Activated Solid-State Switches, A. Rosen and F. J. Zutavern, Eds., Artech House, Boston, 1993, pp. 245-296.

[3] See Proceedings from: 6-8th IEEE Pulsed Power Conf., 1987, 1989, 1991; 18-20th IEEE Power Modulator Symp., 1988, 1990, 1992; SPIE Optically Activated Switching IIII, (vol. 1378, 1632, 1873), 1990, 1992, 1993; and IEEE Trans. Elec. Devices, (vol. 37, 38), 1990, 1992. 
